\title{
Weighted Anisotropic Morrey Spaces Estimates for Anisotropic Maximal Operators
}

\author{
Ferit Gürbüz \\ Department of Mathematics, Faculty of Science, Ankara University \\ Email: feritgurbuz84@hotmail.com
}

\begin{abstract}
The aim of this paper can give weighted anisotropic Morrey Spaces estimates for anisotropic maximal functions.
\end{abstract}

Keywords: Weighted anisotropic Morrey Space, anisotropic maximal function, $A_{p}$ weights

\section{Introduction}

To characterize the regularity of solutions to some partial differential equations(PDEs), Morrey [1] first introduced classical Morrey spaces $M_{p, \lambda}$ which naturally are generalizations of Lebesgue spaces. We also refer to [2],[3] for the latest research on the theory of Morrey spaces associated with harmonic analysis. Next, we recall the definition of weighted Lebesgue spaces. By a "weight" we will mean a non-negative function $w$ that is positive measure a.e. on $\mathbb{R}^{n}$.

Definition 1. (Weighted Lebesgue space) Let $1 \leq p \leq \infty$ and $w$ be a weight function; $w(x) \geq 0$ and $w \in L^{\text {loc }}\left(\mathbb{R}^{n}\right)$, we shall define weighted Lebesgue spaces as

$$
\begin{aligned}
& L_{p}(w) \equiv L_{p}\left(\mathbb{R}^{n}, w\right)=\left\{f:\|f\|_{L_{p, w}}=\left(\int_{\mathbb{R}^{n}}|f(x)|^{p} w(x) d x\right)^{\frac{1}{p}}<\infty\right\}, \quad 1 \leq p<\infty . \\
& L_{\infty, w} \equiv L_{\infty}\left(\mathbb{R}^{n}, w\right)=\left\{f:\|f\|_{L_{\infty, w}}=\underset{x \in \mathbb{R}^{n}}{\operatorname{esssup}}|f(x)| w(x)<\infty\right\} .
\end{aligned}
$$

Then, Komori and Shirai [4] introduced a version of the weighted Morrey space $L_{p, \kappa}(w)$, which is a natural generalization of the weighted Lebesgue space $L_{p}(w)$, and investigated the boundedness of classical operators in harmonic analysis (see [4] for details). Motivated by [4], in this paper we shall introduce the weighted anisotropic Morrey Spaces and investigate the boundedness of the anisotropic maximal functions on this space.

\section{Definition and Notations}

Throughout this paper all notation is standard or will be defined as needed.

Let $\mathbb{R}^{n}$ be the $n$-dimensional Euclidean space of points $x=\left(x_{1}, \ldots, x_{n}\right)$ with norm $\left.|x|=\sum_{i=1}^{n} x_{i}^{2}\right)^{\frac{1}{2}}$, $Q=Q\left(x_{0}, r\right)$ denotes the cube centered at $x_{0}$ with side length $r$. Given a cube $Q$ and $\lambda>0, \lambda Q$ denotes the cube with the same center as $Q$ whose side length is $\lambda$ times that of $Q$. A weight is a locally integrable function on $\mathbb{R}^{n}$ which takes values in $(0, \infty)$ almost everywhere. For a weight function $w$ and a measurable set $E$, we define $w(E)=\int_{E} w(x) d x$, the Lebesgue measure of $E$ by $|E|$ and the characteristic function of $E$ by $\chi_{E}$. Given a weight function $w$, we say that $w$ satisfies the doubling condition if there exists a constant $D>0$ such that for any cube $Q$, we have $w(2 Q) \leq D w(Q)$. When $w$ satisfies this condition, we denote $w \in \Delta_{2}$, for short. 
Let $\mathbb{R}_{0}^{n}=\mathbb{R}^{n} \backslash\{0\}$ and $\mathbb{Z}$ be the set of integer numbers. Let also $a=\left(a_{1}, \ldots, a_{n}\right)$ be a fixed vector from $\mathbb{R}^{n}$ with $a_{i}>0, i=1, \ldots, n$. Consider a real $n \times n$ matrix $A$ with eigenvalues $\lambda_{j}, \operatorname{Re} \lambda_{j}=a_{j}>0$ and let $Q=\operatorname{tr} A$ be its trace. The matrix $A$ determines a one-parameter group $A_{t}=\exp (A \ln t), t>0$ of nonsingular transformations of $\mathbb{R}^{n}$. Denote by $\operatorname{diag}\left\{a_{1}, \ldots, a_{n}\right\}$ the matrix with numbers $a_{1}, \ldots, a_{n}$ on the main diagonal and zero off -diagonal elements and let $a_{\max }=\max _{1 \leq i \leq n} a_{i}$. Associated with the group $A_{t}$ is the $A_{t}$-homogeneous metric $\rho: \mathbb{R}_{0}^{n} \rightarrow \mathbb{R}_{+}, \rho\left(A_{t} x\right)=t \rho(x)$ which is smooth on $\mathbb{R}_{0}^{n}$.

For $x \in \mathbb{R}_{0}^{n}$, let $[x]_{a}$ be a positive solution to the equation $\sum_{i=1}^{n} x_{i}^{2}[x]_{a}^{-2 a_{i}}=1$ and $|x|_{a}=\max _{1 \leq i \leq n}\left|x_{i}\right|^{\frac{1}{a_{i}}}$. Note that $\rho(x)$ is equivalent to $|x|_{a}$, i.e.,

$$
c_{1}|x|_{a} \leq \rho(x) \leq c_{2}|x|_{a} .
$$

For $x \in \mathbb{R}^{n}$ and $r>0$ we define the one-parametric parallelepiped

$$
\begin{aligned}
E(x, t) & =\left\{y \in \mathbb{R}^{n}:|x-y|_{a} \leq t\right\} \\
& =\left\{y \in \mathbb{R}^{n}:\left|y_{i}-x_{i}\right| \leq t^{a_{i}}, i=1, \ldots, n\right\}
\end{aligned}
$$

and by $E=E(\alpha)$ we denote the set of all $E(x, t)$ with $x \in \mathbb{R}^{n}, t>0$. If $a_{1}=\cdots=a_{n}$, then $E(x, t)$ is a cube.

All parallelepipeds are assumed to have their sides parallel to the coordinate axes. $E=E\left(x_{0}, r\right)$ denotes the parallelepiped centered at $x_{0}$ with side length $r^{\alpha_{1}}, \ldots, r^{\alpha_{n}}$ consequently. Given a parallelepiped $E$ and $\lambda>0, \lambda^{a} E$ denotes the parallelepiped with the same center as $E$ whose side length is $(\lambda r)^{a_{1}}, \ldots,(\lambda r)^{a_{n}}$ consequently.

Unless otherwise indicated, the letter $C$ is used for various constants, and may change from one occurrence to another. First we introduce a weighted anisotropic Morrey space.

Definition 2. (Weighted anisotropic Morrey spaces) Let $1 \leq p<\infty, 0 \leq \kappa<1$ and $w$ be a weight. Then a weighted anisotropic Morrey space is defined by

$$
L_{p, \kappa, a}(w):=\left\{f \in L^{l o c}(w):\|f\|_{L_{p, \kappa, a}(w)}<\infty\right\}
$$

where

$$
\|f\|_{L_{p, \kappa, a}(w)}=\sup _{E}\left(\frac{1}{w(E)^{\kappa}} \int_{E}|f(x)|^{p} w(x) d x\right)^{\frac{1}{p}}
$$

and the supremum is taken over all parallelepipeds $E$ on $\mathbb{R}^{n}$. In the case of $a=(1, \ldots, 1)$, we get weighted Morrey spaces $L_{p, \kappa}(w)=L_{p, \kappa, 1}(w)$.

Remark 1. Alternatively, we could define the weighted Morrey spaces with anisotropic balls instead of parallelepipeds. Hence we shall use these two definitions of weighted anisotropic Morrey spaces appropriate to calculation. Also, we could define the weighted Morrey spaces with cubes instead of parallelepipeds. Moreover, when $a_{1}=\cdots=a_{n}=1$, then $\rho(x)=|x|$ and $\alpha=n$. In this case, weighted anisotropic Morrey spaces is reduced to the weighted Morrey space:

$$
\|f\|_{L_{p, \kappa, a}(w)}=\|f\|_{L_{p, \kappa}(w)} .
$$

Thus from this viewpoint, Theorem 4 and Theorem 4 below improve the corresponding results in [3],[4]. In other words, in this paper our goal is to extend results in [3],[4].

Remark 2. (1) If $w \equiv 1$ and $\kappa=\lambda / n$ with $0<\lambda<n$, then $L_{p, \kappa}(w)=L_{p, \lambda}\left(\mathbb{R}^{n}\right)$ is the classical Morrey spaces. If $w \equiv 1$ and $\kappa=\frac{\lambda}{|a|}$ with $0<\lambda<|a|,|a|=a_{1}+\cdots+a_{n}$, then $L_{p, \kappa, a}(w)=L_{p, \lambda, a}(w)$ the anisotropic Morrey spaces. (2) Let $w \in \Delta_{2}$. If $\kappa=0$, then $L_{p, 0}(w)=L_{p}(w)$ is the weighted Lebesgue spaces. If $\kappa=1$, then $L_{p, 1}(w)=L_{\infty}(w)$ by the Lebesgue differentiation theorem with respect to $w$ (see 
[5]). (3) In the one-dimensional case, let a weight $w(x)=|x|^{\alpha}$ for some $-\frac{1}{2}<\alpha<0$ and a function $f(x)=\chi_{(0,1)}|x|^{-\frac{1}{2}}$. Then $f \in L^{1, \frac{\alpha+\frac{1}{2}}{\alpha+1}}(w) \backslash L^{2(\alpha+1)}(w)$.

Let $f \in L^{\text {loc }}\left(\mathbb{R}^{n}\right)$. The anisotropic maximal function $M f$ and the sharp maximal function $f^{\#}$ are defined by

$$
M f(x)=\sup _{t>0}|E(x, t)|^{-1} \int_{E(x, t)}|f(y)| d y
$$

and

$$
f^{\#}(x)=\sup _{t>0}|E(x, t)|^{-1} \int_{E(x, t)}\left|f(y)-f_{E(x, t)}\right| d y,
$$

where $f_{E(x, t)}=|E(x, t)|^{-1} \int_{E(x, t)}|f(y)| d y$.

If $a_{1}=\cdots=a_{n}$, then $E(x, t)$ is a cube and $M f$ becomes the usual Hardy-Littlewood maximal function. For $r>0$, we denote $M_{r} f(x)$ by $\left(M|f|^{r}(x)\right)^{\frac{1}{r}}$.

Let $w$ be a weight. $M_{w}$ denotes the anisotropic maximal operator with respect to the measure $w(x) d x$ defined by

$$
M_{w} f(x)=\sup _{E} \frac{1}{w(E)} \int_{E}|f(y)| w(y) d y
$$

Here and after, $A_{p}$ denotes the Muckenhoupt classes (see [3]). In other words, let $1<p<\infty$. One says that $w(x) \in A_{p}\left(\mathbb{R}^{n}\right)$, if the following condition holds:

$$
\begin{aligned}
{[w]_{A_{p}} } & :=\sup _{E}[w]_{A_{p}(E)} \\
& =\sup _{E}\left(\frac{1}{|E|} \int_{E} w(x) d x\right)\left(\frac{1}{|E|} \int_{E} w(x)^{1-p^{\prime}} d x\right)^{p-1}<\infty,
\end{aligned}
$$

where the supremum is taken with respect to any parallelepiped $E$ and $p^{\prime}=\frac{p}{p-1}$. The condition (2) is called the $A_{p}$-condition, and the weights which satisfy it are called $A_{p}$-weights. The property of the $A_{p}$-weights implies that generally speaking, we should check whether a weight $w$ satisfies an $A_{p^{-}}$-condition or not. The expression $[w]_{A_{p}}$ is also called characteristic constant of $w$. For the case $p=1, w \in A_{1}\left(\mathbb{R}^{n}\right)$ if

$$
\frac{1}{|B|} \int_{B} w(x) d x \leq C \inf _{x \in B} w(x),
$$

and the infimum of these constant $C$ is called the $A_{1}$ constant of $w$ and denoted by $[w]_{A_{1}}$. Since the $A_{p}$ classes are increasing with respect to $p$, the $A_{\infty}$ class of weights is defined in a natural way by $A_{\infty}\left(\mathbb{R}^{n}\right)=\bigcup_{1 \leq p<\infty} A_{p}\left(\mathbb{R}^{n}\right)$ and the $A_{\infty}$ constant of $w(x) \in A_{\infty}\left(\mathbb{R}^{n}\right)$ is the smallest of the infimum of the $A_{p}$ constant such that $w(x) \in A_{p}\left(\mathbb{R}^{n}\right),[w]_{A_{\infty}}=\inf _{1 \leq p<\infty}[w]_{A_{p}}$ and $[w]_{A_{\infty}} \leq[w]_{A_{p}}$.

It is easy to verify that, $\rho(x)^{\alpha} \in A_{p}$ if and only if $-|a|<\alpha<|a|(p-1)$ for $1<p<\infty$ and $\rho(x)^{\alpha} \in A_{1}$ if and only if $-|a|<\alpha \leq 0$.

\section{Lemmas and Well Known Results}

In this section, we shall prove some lemmas and describe the well-known result about the weighted $L_{p}$ spaces.

Theorem 1. ([[6], Theorem 2.6, p. 146]) If $1<p<\infty$ and $w \in \Delta_{2}$, then the operator $M_{w}$ is bounded on $L_{p}(w)$.

The next lemma plays an important role in our proofs of theorems. We say that $w$ satisfies the reverse doubling condition if $w$ has the property (3) of the following lemma. 
Lemma 1. ([4])If $w \in \Delta_{2}$, then there exists a constant $D_{1}>1$ such that

$$
w(2 E) \geq D_{1} w(E) .
$$

Proof. Fix a parallelepiped $E=E\left(x_{0}, r\right)$. Then we can choose a parallelepiped $R \subset 2 E$ with side length $\frac{r}{2}$ which is disjoint from the parallelepiped $E$. Hence

$$
w(E)+w(R) \leq w(2 E) .
$$

On the other hand, since $E \subset 5 R$ we have $w(E) \leq w(5 R) \leq D^{3} w(R)$, where $D$ is a doubling constant. Therefore we have

$$
w(E)+\frac{w(E)}{D^{3}} \leq w(2 E) .
$$

Lemma 2. ([7]) The following statements hold:

(1) If $w \in A_{p}$ for some $1 \leq p<\infty$, then $w \in \Delta_{2}$. Moreover, for all $\lambda>1$ we have

$$
w(\lambda E) \leq \lambda^{n p}[w]_{A_{p}} w(E) .
$$

(2) Let $w \in A_{p}$ for some $1 \leq p<\infty$. Then we have

$$
M f(x) \leq[w]_{A_{p}}^{\frac{1}{p}}\left(M_{w}\left(|f|^{p}\right)(x)\right)^{\frac{1}{p}} .
$$

Proof. (1) Let $w \in A_{p}$ for some $1 \leq p<\infty$ and $\lambda>1$. Then

$$
\begin{aligned}
\frac{w(\lambda E)}{w(E)} & =\left(\frac{|\lambda E|}{|E|}\right)^{p} \frac{[w]_{A_{p}}(\lambda E)}{[w]_{A_{p}}(E)} \frac{\left(\int_{E} w(x)^{1-p^{\prime}} d x\right)^{p-1}}{\left(\int_{\lambda E} w(x)^{1-p^{\prime}} d x\right)^{p-1}} \\
& \leq \lambda^{n p}[w]_{A_{p}} .
\end{aligned}
$$

(2) Let $w \in A_{p}$ for some $1 \leq p<\infty$. Applying the Hölder's inequality, we get

$$
\begin{aligned}
M f(x) & =\sup _{E} \frac{1}{|E|} \int_{E}|f(x)| d x \\
& \leq \sup _{E} \frac{1}{|E|}\left(\int_{E}|f(x)|^{p} w(x) d x\right)^{\frac{1}{p}}\left(\int_{E} w(x)^{1-p^{\prime}} d x\right)^{\frac{1}{p^{\prime}}} \\
& \left.\left.=\sup _{E}\left(\frac{1}{w(E)} \int_{E}|f(x)|^{p} w(x) d x\right)^{\frac{1}{p}}\left(\int_{E} w(x)^{1-p^{\prime}} d x\right)^{\frac{1}{p^{\prime}}}\left(\frac{w(E)}{|E|}\right)^{\frac{1}{p}}\right)_{E} \int_{E}\left(\frac{1}{|E|} \int_{E} w(x) d x\right)\left(\int_{E} w(x)^{1-p^{\prime}} d x\right)^{p-1}\right)^{\frac{1}{p}} \\
& \left.=\sup _{E} \frac{1}{w(E)}\right)^{\frac{1}{p}}\left(M_{w}\left(|f|^{p}\right)(x)\right)^{\frac{1}{p}} .
\end{aligned}
$$

\section{Anisotropic Maximal Function}

In this section, we shall state the boundedness of the anisotropic maximal operators on weighted anisotropic Morrey Spaces. 
Theorem 2. (Our main result) If $1<p<\infty, 0<\kappa<1$ and $w \in \Delta_{2}$, then the operator $M_{w}$ is bounded on $L_{p, \kappa, a}(w)$.

Proof. Fix a parallelepiped $E \subset \mathbb{R}^{n}$. We decompose $f=f_{1}+f_{2}$, where $f_{1}=f \chi_{3 E}$. Since $M_{w}$ is a sublinear operator, we have

$$
\begin{aligned}
& \left(\int_{E} M_{w} f(x)^{p} w(x) d x\right)^{\frac{1}{p}} \leq\left(\int_{E} M_{w} f_{1}(x)^{p} w(x) d x\right)^{\frac{1}{p}}+\left(\int_{E} M_{w} f_{2}(x)^{p} w(x) d x\right)^{\frac{1}{p}} \\
& =I+I I .
\end{aligned}
$$

For the term $I$, since $M_{w}$ is bounded on $L_{p}(w)$ (see Theorem 3 ), we obtain

$$
\begin{aligned}
I & \leq\left(\int_{E} M_{w} f_{1}(x)^{p} w(x) d x\right)^{\frac{1}{p}} \leq C\left(\int_{3 E}|f(x)|^{p} w(x) d x\right)^{\frac{1}{p}} \\
& \leq C\|f\|_{L_{p, \kappa, a}(w)} w(E)^{\frac{\kappa}{p}} .
\end{aligned}
$$

Next we estimate the term $I I$. By simple geometric observation, we have for any $x \in E$, note that for all $\tilde{E}$ such that $x \in \tilde{E}, \tilde{E} \cap(3 E)^{c} \neq \emptyset$ there exists $R$ such that $E \subset 3 R$ and $\frac{1}{3} R \subset \tilde{E} \subset R$.

Then $w(\tilde{E}) \geq w\left(\frac{1}{3} R\right) \geq \frac{1}{D_{1}} w(R)$ and

$$
M_{w} f_{2}(x) \leq D_{1} \sup _{R: E \subset 3 R} \frac{1}{w(R)} \int_{R}|f(y)| w(y) d y .
$$

Note that

$$
\begin{aligned}
& \frac{1}{w(R)} \int_{R}|f(y)| w(y) d y \leq \frac{1}{w(R)}\left(\int_{R}|f(y)|^{p} w(y) d y\right)^{\frac{1}{p}}\left(\int_{R} w(y) d y\right)^{\frac{1}{p^{\prime}}} \\
& \leq \frac{1}{w(R)^{1-\frac{1}{p^{\prime}}}}\left(\int_{R}|f(y)|^{p} w(y) d y\right)^{\frac{1}{p}}=\frac{1}{w(R)^{\frac{1}{p}}}\left(\frac{1}{w(R)^{\kappa}} \int_{R}|f(y)|^{p} w(y) d y\right)^{\frac{1}{p}} w(R)^{\frac{\kappa}{p}} \\
& \leq\left(\frac{1}{w(R)^{\kappa}} \int_{R}|f(y)|^{p} w(y) d y\right)^{\frac{1}{p}} w(R)^{\frac{\kappa-1}{p}} \leq C\|f\|_{L_{p, \kappa, a}(w)} w(R)^{\frac{\kappa-1}{p}},
\end{aligned}
$$

if $E \subset 3 R$. So we obtain

$$
\begin{aligned}
I I & =\left(\int_{E} M_{w} f_{2}(x)^{p} w(x) d x\right)^{\frac{1}{p}} \leq\left(\int_{E}\left[\sup _{x \in R} \frac{1}{w(R)} \int_{R}|f(y)| w(y) d y\right]^{p} w(x) d x\right)^{\frac{1}{p}} \\
& \leq\left(\int_{E}\left[c\|f\|_{L_{p, \kappa, a}(w)} w(E)^{\frac{\kappa-1}{p}}\right]^{p} w(x) d x\right)^{\frac{1}{p}} \leq c\|f\|_{L_{p, \kappa, a}(w)} w(E)^{\frac{\kappa-1}{p}}\left(\int_{E} w(x) d x\right)^{\frac{1}{p}} \\
& =c\|f\|_{L_{p, \kappa, a}(w)} w(E)^{\frac{\kappa}{p}}
\end{aligned}
$$

Therefore

$$
I I \leq C\|f\|_{L_{p, \kappa, a}(w)} w(E)^{\frac{\kappa}{p}} .
$$

This completes the proof. 
Corollary 1. ([4], Theorem 3.1) If $1<p<\infty, 0<\kappa<1$ and and $w \in \Delta_{2}$, then the operator $M_{w}$ is bounded on $L_{p, \kappa}(w)$.

Theorem 3. (Our main result) If $1<p<\infty, 0<\kappa<1$ and $w \in A_{p}$, then the anisotropic maximal operator $M$ is bounded on $L_{p, \kappa, a}(w)$.

If $p=1,0<\kappa<1$ and $w \in A_{1}$, for all $t>0$ and any parallelepiped $E$,

$$
w(x \in E: M f(x)>t) \leq \frac{C}{t}\|f\|_{L_{1, \kappa, a}(w)} w(E)^{\kappa} .
$$

Proof. Let $1<p<\infty$. By the reverse Hölder inequality (see [8]), there exists $1<r<p$ such that $w \in A_{r}$. Hence it follows from Lemma 3 (2) and Theorem 4 that

$$
\begin{aligned}
& \left(\frac{1}{w(E)^{\kappa}} \int_{E} M f(x)^{p} w(x) d x\right)^{\frac{1}{p}} \leq C\left(\frac{1}{w(E)^{\kappa}} \int_{E} M_{w}\left(|f|^{r}\right)(x)^{\frac{p}{r}} w(x) d x\right)^{\frac{1}{p}} \\
& \left.\leq C\left\|M_{w}\left(|f|^{r}\right)\right\|_{L_{\frac{p}{r}, \kappa, a}^{\frac{1}{r}}(w)} \leq C\left\||f|^{r}\right\|_{L_{\frac{p}{r}, \kappa, a}^{\frac{1}{r}}(w)}^{\frac{r}{p}}\right]^{\frac{1}{r}}=C\|f\|_{L_{p, \kappa, a}(w)} . \\
& =c\left[\sup _{E}\left(\frac{1}{w(E)^{\kappa}} \int_{E}|f(x)|^{r\left(\frac{p}{r}\right)} w(x) d x\right)^{2}\right.
\end{aligned}
$$

When $p=1$, we use the Fefferman-Stein maximal inequality

$$
\int_{\{x: M f(x)>t\}} \varphi(x) d x \leq \frac{C}{t} \int_{\mathbb{R}^{n}}|f(x)|\left(M_{\varphi}\right)(x) d x
$$

for any functions $f$ and $\varphi \geq 0$ (see [9],[6]).

Fix a parallelepiped $E=E\left(x_{0}, r\right)$. Put $\varphi(x)=w(x) \chi_{E}(x)$. Then we have

$$
\begin{aligned}
& \int_{\{x: M f(x)>t\}} \chi_{E}(x) w(x) d x \leq \frac{C}{t} \int_{\mathbb{R}^{n}}|f(x)| M\left(w \chi_{E}\right)(x) d x \\
= & \frac{C}{t}\left(\int_{3 E}+\int_{(3 E)^{c}}\right)=\frac{C}{t}\{I+I I\}
\end{aligned}
$$

for all $t>0$.

We now estimate the term $I$. Since $w \in A_{1}$, it follows that

$$
M\left(w \chi_{E}\right)(x) \leq M(w)(x) \leq C w(x) .
$$

So it follows that

$$
I \leq C w(3 E)^{\kappa}\|f\|_{L_{1, \kappa, a}(w)} \leq C w(E)^{\kappa}\|f\|_{L_{1, \kappa, a}(w)} .
$$

To estimate the term $I I$, we consider the form

$$
\frac{1}{|R|} \int_{R \cap E} w(y) d y
$$

for $x \in(3 E)^{c} \cap R$ and $R \cap E \neq \emptyset$. By simple geometric observation, we have

$$
\frac{1}{|R|} \int_{R \cap E} w(y) d y \leq C_{n}\left(\frac{1}{\left|x-x_{0}\right|_{a}^{|a|}} \int_{E} w(y) d y\right) \leq C_{n}\left|x-x_{0}\right|_{a}^{-|a|} w(E) .
$$


Therefore we obtain

$$
M\left(w \chi_{E}\right)(x) \leq C_{n}\left|x-x_{0}\right|_{a}^{-|a|} w(E) .
$$

Since $w \in A_{1}$, we have $w \in \Delta_{2}$ by Lemma 3 (1). Using Lemma 3, we have $w(3 E) \geq w(2 E) \geq D_{1} w(E)$ with $D_{1}>1$. Thus we can estimate the term $I I$ as follows:

$$
\begin{aligned}
I I & \leq C w(E) \int_{(3 E)^{c}} \frac{|f(x)|}{\left|x-x_{0}\right|_{a}^{|a|}} d x=C \sum_{j=3_{3^{j+1}}}^{\infty} \int_{E \backslash\left(3^{j} E\right)} \frac{|f(x)|}{\left|x-x_{0}\right|_{a}^{|a|}} d x \\
& \leq C w(E) \sum_{j=1}^{\infty} \frac{1}{\left|3^{j} E\right|} \int_{3^{j+1} E}|f(x)| d x \\
& \leq C w(E) \sum_{j=1}^{\infty} \frac{1}{\left|3^{j} E\right|}\left(\operatorname{esssup}_{x \in 3^{j+1} E} \frac{1}{w(x)}\right) \int_{3^{j+1} E}|f(x)| w(x) d x \\
& =C w(E) \sum_{j=1}^{\infty} \frac{1}{\left|3^{j} E\right|} \frac{\left|3^{j+1} E\right|}{w\left(3^{j+1} E\right)} \int_{3^{j+1} E}|f(x)| w(x) d x \\
& =C w(E)^{\kappa} \sum_{j=1}^{\infty} \frac{w(E)^{1-\kappa}}{w\left(3^{j+1} E\right)^{1-\kappa}} \frac{1}{w\left(3^{j+1} E\right)^{\kappa}} \int_{3^{j+1} E}|f(x)| w(x) d x \\
& \leq C w(E)^{\kappa}\|f\|_{L_{1, \kappa, a}(w)} \sum_{j=1}^{\infty} \frac{w(E)^{1-\kappa}}{w\left(3^{j+1} E\right)^{1-\kappa}} \leq C w(E)^{\kappa}\|f\|_{L_{1, \kappa, a}(w)}
\end{aligned}
$$

The last series converges since the reverse doubling constant is larger than one (see Lemma 3). This completes the proof.

Corollary 2. ([4], Theorem 3.2) If $1<p<\infty, 0<\kappa<1$ and $w \in A_{p}$, then the Hardy-Littlewood maximal operator $M$ is bounded on $L_{p, \kappa}(w)$. If $p=1,0<\kappa<1$ and $w \in A_{1}$, for all $t>0$ and any cube $Q$,

$$
w(\{x \in Q: M f(x)>t\}) \leq \frac{C}{t}\|f\|_{L_{1, \kappa}(w)} w(Q)^{\kappa} .
$$

Corollary 3. ([3], Theorem 2) Let $1<p<\infty, 0 \leq \kappa<1$ and $w \in A_{p}$. If $A$ is a (not necessarily quasi-homogenous) pseudo-differential operator of order 0 , then the operator $A$ is bounded on $L_{p, \kappa}(w)$.

Corollary 4. ([3], Corollary 1) Let $m \in \mathbb{N}, 1 \leq j \leq n, 1<p<\infty, 0 \leq \kappa<1, w \in A_{p}$ and $a(x, \xi)=$ $\frac{\xi_{j}^{m}}{\left(1+|\xi|^{2}\right)^{\frac{m}{2}}}$. Then

$$
\left\|F^{-1}\left(\xi_{j}^{m}\left(1+|\xi|^{2}\right)^{-\frac{m}{2}} F f\right)\right\|_{L_{p, \kappa}(w)} \leq C\|f\|_{L_{p, \kappa}(w)} .
$$

\section{References}

1. C. B. Morrey, "On the solutions of quasi-linear elliptic partial differential equations," Trans. Amer. Math. Soc., vol. 43, pp. 126-166, 1938.

2. D. Adams, Morrey spaces. Lecture Notes in Applied and Numerical Harmonic Analysis. Birkhäuser/Springer, 2015 .

3. F. Gurbuz, "Weighted morrey and weighted fractional sobolev-morrey spaces estimates for a large class of pseudo-differential operators with smooth symbols," J. Pseudo-Differ. Oper. Appl., vol. 7, pp. 595-607, 2016.

4. Y. Komori and S. Shirai, "Weighted morrey spaces and a singular integral operator," Math. Nachr., vol. 282, pp. 219-231, 2009.

5. W. Rudin, Real and complex analysis. McGraw-Hill, New York, 1966.

6. R. A. Hunt, B. Muchenoupt, and R. L. Wheeden, "Weighted norm inequalities for the conjugate function and hilbert transform," Trans. Amer. Math. Soc., vol. 176, pp. 227-251, 1973. 
7. V. M. Kokilashvili and J. Rakosnik, "Weighted inequalities for anisotropic maximal functions," Casopis pro pestovani matematiky, vol. 110.

8. R. R. Coifman and C. Fefferman, "Weighted norm inequalities for maximal functions and singular integrals," Studia Math., vol. 51, pp. 241-250, 1974.

9. C. Fefferman and E. M. Stein, "Some maximal inequalities," Amer. J. Math., vol. 93, pp. 107-115, 1971. 